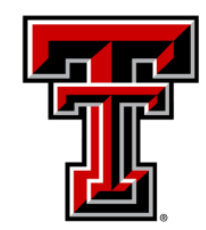

TEXAS TECH UNIVERSITY

Libraries"

\title{
SOLUTIONS FOR LATENT GROWTH MODELING FOLLOWING COVID-19- RELATED DISCONTINUITIES IN CHANGE AND DISRUPTIONS IN LONGITUDINAL DATA COLLECTION
}

\section{The Texas Tech community has made this publication openly available. Please share how this access benefits you. Your story matters to us.}

\begin{tabular}{|l|l|}
\hline Citation & $\begin{array}{l}\text { Rioux, C., Stickley, Z. L., \& Little, T. D. (2021). Solutions for latent } \\
\text { growth modeling following COVID-19-related discontinuities in } \\
\text { change and disruptions in longitudinal data collection. International } \\
\text { Journal of Behavioral Development. } \\
\text { https://doi.org/10.1177/01650254211031631 }\end{array}$ \\
\hline Citable Link & $\underline{\text { https://hdl.handle.net/2346/87456 }}$ \\
\hline Terms of Use & $\underline{\text { CC BY-NC-ND 4.0 }}$ \\
\hline
\end{tabular}


Accepted manuscript published in International Journal of Behavioral Development https://doi.org/10.1177/01650254211031631

Solutions for latent growth modeling following COVID-19-related discontinuities in change and disruptions in longitudinal data collection

\author{
Charlie Rioux ${ }^{1}$, Zachary L. Stickley ${ }^{1}$ and Todd D. Little ${ }^{1,2}$ \\ ${ }^{1}$ Department of Educational Leadership and Psychology, Texas Tech University \\ ${ }^{2}$ Optentia Research Focus Area, North-West University, Vanderbijlpark, South Africa
}

\begin{abstract}
Author note
Acknowledgements: This article was supported by the Canadian Institute of Health Research through a fellowship to CR and the Fonds de Recherche du Québec - Santé through a fellowship to CR.

Correspondence concerning this article should be addressed to Dr. Charlie Rioux, charlie.rioux@ttu.edu.
\end{abstract}




\begin{abstract}
Following the onset of the novel coronavirus disease 2019 (COVID-19) pandemic, daily life significantly changed for the population. Accordingly, researchers interested in examining patterns of change over time may now face discontinuities around the pandemic. Researchers collecting in-person longitudinal data also had to cancel or delay data collection waves, further complicating analyses. Accordingly, the purpose of this article is to aid researchers aiming to examine latent growth models (LGM) in analysing their data following COVID-19. An overview of basic LGM notions, LGMs with discontinuities, and solutions for studies that had to cancel or delay data collection waves are discussed and exemplified using simulated data. Syntax for R and Mplus is available to readers in online supplemental materials.
\end{abstract}

Keywords: growth curve, discontinuity, piecewise, analysis, missing data, SARS-COV-2 


\section{Solutions for latent growth modeling following COVID-19-related discontinuities in change and disruptions in longitudinal data collection}

Latent growth models (LGMs) are frequently used when a study aims to examine intraindividual patterns of change over time and inter-individual variability in these patterns of change. Following the onset of the novel coronavirus disease 2019 (COVID-19) pandemic, these patterns of change may have been affected by discontinuities, and some longitudinal studies may have had to cancel or delay data collection waves. Many researchers are familiar with continuous growth models (linear and non-linear such as quadratic) and longitudinal studies with equidistant data collection waves, but may be less familiar with more advanced applications of these techniques and are unsure of how to account for both pandemic-related discontinuities in developmental processes and disruptions in data collection.

Accordingly, in the present article, we aim to aid researchers by presenting LGMs that can be flexibly applied to datasets for which data collection was ongoing before and during COVID-19. First, a refresher on latent growth modeling is provided. Then, LGMs that can estimate discontinuities in growth patterns are presented. Finally, some solutions for studies that had to cancel or delay data collection waves are discussed.

\section{Refresher on latent growth modeling}

Latent growth modeling is used to study change over time when participants from a studied population are expected to have a similar growth trajectory (e.g., linear) with variability in terms of initial levels and rate of change. In a first step, on which the present paper will focus, researchers can estimate unconditional growth models which allow describing mean growth in the sample with a minimum of two latent factors, i.e., an intercept and a slope. In a second step, researchers may enter predictors or outcomes of these latent growth factors. While the present 
article centers around LGMs implemented in a structural equation modeling framework, growth models can also be estimated using multilevel regression approaches, see Grimm et al. (2016) and Singer and Willett (2003).

The linear LGM is one of the most commonly fit models in applied research and requires at least three repeated assessments of the construct of interest. It consists of two latent variables; an intercept capturing levels at a chosen time point and a slope capturing rates of change. While the intercept is most commonly centered at the first time point, which is why it is often referred to as capturing initial levels, it can be centered at any time point if they are of more interest for the research question.

A path diagram of the factor model underlying the linear LGM is presented in Figure 1. Following SEM conventions, squares represent observed variables, circles represent latent variables, triangles represent unit constants used to estimate means, one-headed arrows represent directional relationships (e.g., regression) and double-headed arrows represent nondirectional relationships (e.g., correlations). This diagram clearly shows that the intercept and slope are latent factors, which are conceptualized as predicting answers on the observed indicators at each time point. To model a linear growth with equidistant measurement occasions, all intercept construct loadings would be fixed to 1.0, and the loadings of the slope construct would be fixed to a linear pattern (e.g., 0.0, 1.0, and 2.0). The mean and variance of the intercept and slope as well as the covariance between the two would be allowed to freely estimate. The slope loading of 0.0 centers the intercept, which is centered here at the first time point.

The present article presents different patterns of change that may be useful to estimate pandemic-related effects, as well as modifications to these models following disruptions in data collection. All models presented will expand on the linear model and will include one or several 
linear slope(s). Note that non-linear change (e.g., quadratic) can be modelled in SEM and one or several slopes in all models presented could be estimated as non-linear slopes. For details on modeling non-linear slopes, see Grimm et al. (2016). Models and solutions are based on the frequently planned longitudinal data collection with equidistant data collection waves that are common among participants. For models with individually varying assessment times, see timeunstructured models (Coulombe et al., 2016; Mehta \& West, 2000).

In an SEM framework, observed variables or latent factors can be used as indicators in LGMs, the later being referred to as a latent-variable growth model or a curve-of-factors model. For such a model, confirmatory factor analysis (CFA) is first used to estimate a latent factor of the construct of interest at each time point. Longitudinal measurement invariance testing should then be conducted to examine whether the measurement structure of the latent factor is stable over time (van de Schoot et al., 2015; Widaman et al., 2010). If a latent factor is invariant, the same construct is assessed using the same metric across time, and thus growth patterns can be attributed to actual change rather than measurement artifacts. Invariance is an assumption in all growth models but can only be formally tested by examining invariance of latent factors. The present article will use examples with observed indicators since the focus is to present variations in estimating the pattern of growth; that is, the shape of the growth trajectories as interpreted through the latent growth factors (e.g., intercept and slope factors). The growth specification of all these models can be used with latent factor indicators. For details on testing longitudinal invariance and fitting latent-variable growth models, see Isiordia and Ferrer (2018) and Little (2013), chapters 5 and 8.

Linear LGM, Example 1. Throughout this article, we use simulated data to illustrate applications of the methods described. For all examples, the data were simulated using a model- 
based data simulation technique. Population models were specified in the lavaan package 0.6-7 (Rosseel, 2012) in R version 4.0.3 (R Core Team, 2020) using the RStudio interface version 1.4.1103 (RStudio Team, 2020). The simulation R script is available in online supplemental material. After simulating the data, analytical models were run in both lavaan 0.6-7 (Rosseel, 2012) and Mplus version 8.3 (Muthén \& Muthén, 1998-2017). For all examples in this paper, syntax is provided in online supplemental material for estimating the models in R (lavaan) or Mplus.

For illustrative purposes and based on results from Skibbe et al. (2012), our data simulates a literacy measure of passage comprehension using a standardized scale centered at 500 points, representing average abilities in Grade 5. In our simulated examples, 500 student participants are followed from one year before kindergarten (Pre-K) to Grade 1 over six biannual assessments, from October 2018 to April 2021.

Under linear growth conditions (see Figure 2), these data would be modeled by fixing all intercept construct loadings to 1 and fixing the loadings of the slope construct to a linear pattern from 0 to 5. Models in lavaan and Mplus return the same results. Three categories of parameters are important in the interpretation of LGM results: the mean of the latent factors, the variance of the latent factors, and the covariance between latent factors.

Growth factor means were significantly different from zero and estimated at 423.03 (SE $=.17, \mathrm{p}<.001)$ for the intercept and $17.97(\mathrm{SE}=.10, \mathrm{p}<.001)$ for the slope. These values indicate an average level in passage comprehension of 423.03 points in the fall (October) of Pre$\mathrm{K}$ (the first time point, at which the intercept is centered), with an average increase of 17.97 points per 6 months (because an increase of 1 in slope loadings corresponds to 6 months). The means of the intercept and slope factors can be used to plot values at each time point and draw 
the average growth trajectory, as seen in Figure 3. This is done by adding the mean value of all growth factors (here, the intercept and the slope), each multiplied by their respective loading for that time point. An excel spreadsheet to aid in calculating estimated values and drawing growth trajectories for the different models presented in the paper is provided in online supplemental material.

Variance was also significant for both the intercept $(14.34, \mathrm{SE}=.92, \mathrm{p}<.001)$ and the slope $(4.84, \mathrm{SE}=.31, \mathrm{p}<.001)$. Significant variance indicates that participants vary around the mean value and is a necessary component when research objectives aim to examine predictors or outcomes of the growth factors. Finally, regarding covariances, the intercept and the slope were significantly and positively correlated $(r=.13, \mathrm{SE}=.38, \mathrm{p}=.004)$, indicating that higher initial levels of passage comprehension were associated with steeper increases in passage comprehension. Variances and covariances are important parameters for interpretation in substantive developmental research but are not reported in the remainder of models presented in this paper as the focus is on the shape of growth trajectories, which is interpreted using growth factor means. Table 1 provides an overview of the models presented in the remainder of this article.

\section{Latent growth models to estimate pandemic-related discontinuities in development}

Considering that the pandemic affected most aspects of daily life, for many constructs of interest the growth pattern demonstrated by the data may have been affected by the pandemic. For example, the pandemic and shutdowns were found to have potential impacts on physical health (Lange et al., 2020; Pellegrini et al., 2020) and mental health (Rajkumar, 2020; TomfohrMadsen et al., 2021), may affect parenting stress and practices (Marchetti et al., 2020; Roos et al., 2021), and school closures could have impacts on children's and adolescents' education, 
nutrition, social development and psychosocial support (Gupta \& Jawanda, 2020; Masonbrink \& Hurley, 2020). Accordingly, researchers may find that an expected growth pattern (e.g., linear) is not supported by the data because the data post-pandemic onset do not behave the same as the pre-pandemic data. This effect should not be seen as a problem, but rather as an opportunity to examine how development occurred both before and during (and potentially after) the pandemic.

Discontinuity models, where an event alters the growth trajectory, may be particularly relevant to the examination of changes in growth patterns following the onset of the pandemic. Discontinuity LGMs test similar hypotheses as quasi-experimental interrupted times series regressions (Bernal et al., 2017). Specifically, discontinuity LGMs would examine whether a growth trajectory shifted in level and/or slope after pandemic onset by using the timing of the pandemic to inform the analysis, yielding three possible discontinuity models that are presented below. For more resources about discontinuity models, including their estimation in multilevel modeling, see Grimm and Marcoulides (2016); Peugh et al. (2020); and Singer and Willett (2003).

\section{Slope discontinuity latent growth model}

If the pandemic only influences the rate of change, this would be captured using a slope discontinuity LGM, also known as spline or piecewise LGM (Chou et al., 2004; Flora, 2008; Kohli \& Harring, 2013). This model tests hypotheses related to growth patterns around a knot (i.e., turning point). Accordingly, this model would estimate a slope before and after pandemic onset, with a turning point at pandemic onset. In terms of data, a minimum of two unique time points per slope is required. Accordingly, if the knot is at the middle point of the LGM and is centered at an assessment (see Slope discontinuity, Example 2), a minimum of five time points is required. If the knot is centered between two assessments, (see example in Cancelled wave, 
Example 6 below), a minimum of four time points is required. If the knot point is not at the middle point of the LGM, more time points will be required (i.e., at least two before and after the knot point).

Slope discontinuity, Example 2. In our hypothetical example (see Linear LGM, Example 1), students were followed bi-annually from October 2018 to April 2021, with pandemic onset around the time of the April 2020 assessment (fourth of six assessments). For the current example, our model simulates the hypothesis that passage comprehension scores continue to increase after the onset of the pandemic, but at a slower pace. To examine a slope discontinuity model with two linear slopes (see Figure 4), the knot is estimated like an intercept centered using a common 0 loading for the two slopes. Here, both slopes have a loading of 0 for the April 2020 assessment. The loadings of the first slope construct, which is four time points from the first assessment to the knot, are constrained to a linear pattern of -3 to 0 , with zeros after the knot. Similarly, the loadings of the second slope construct, which is three time points from the knot to the last time point, are constrained to a linear pattern of 0 to 2 , with zeros before the knot. Models in lavaan and Mplus return the same results. Growth curve factor means were significantly different from zero and estimated at $477.00(\mathrm{SE}=.36, \mathrm{p}<.001)$ for the knot/intercept, $18.03(\mathrm{SE}=.10, \mathrm{p}<.001)$ for the first slope and $8.93(\mathrm{SE}=.13, \mathrm{p}<.001)$ for the second slope. These values indicate, as shown in Figure 5, an average level of passage comprehension of 477.00 points in April of kindergarten, and that passage comprehension increased on average by 18.03 points per six months before the pandemic, and by 8.93 points per six months after pandemic onset. As can be seen, the estimated values for the first slope are similar to the linear model above, but the growth pattern changes after pandemic onset, with the rate of growth in passage comprehension being slower after April 2020 compared to before. 


\section{Level discontinuity latent growth models}

While the pandemic could alter rates of change in a construct, it could also be associated with a sudden increase or decrease at pandemic onset, with following rates of change (slope) being equivalent to pre-pandemic rates. This bump would be captured by a level discontinuity model, which estimates whether an event (e.g., onset of pandemic) is associated with changes in levels, but with slope estimates being the same before and after the event. This model is sometimes referred to as an intercept discontinuity model, with the event being conceptualized as a new intercept fitted to later time points. In terms of data, a minimum of three assessments is required to estimate an intercept, slope, and bump.

Level discontinuity, Example 3. In this example, our model simulates the hypothesis that sudden shifts in everyday routines at pandemic onset were associated with an abrupt drop in passage comprehension test scores, with scores then following the same increasing trajectory as before the pandemic. To examine a level discontinuity model with a linear slope and the event occurring at the fourth of six assessments (see Figure 6), the loadings of the intercept and slope are constrained in the same manner as for the linear LGM, and a third factor is added to capture the effect of the event (COVID) on the level of the construct. This third factor loads only on the indicators occurring after the event, with all loadings constrained to 1. Models in lavaan and Mplus return the same results. Growth factor means were significantly different from zero and estimated at $423.03(\mathrm{SE}=.17, \mathrm{p}<.001)$ for the intercept, $17.96(\mathrm{SE}=.10, \mathrm{p}<.001)$ for the slope, and $-24.96(\mathrm{SE}=.07, \mathrm{p}<.001)$ for the event level. These values indicate, as shown in Figure 7, an average level in passage comprehension of 423.03 points in October of Pre-K, with an average increase of 17.96 points per six months both pre- and post-pandemic onset, but a drop of 7.00 points at pandemic onset. Thus, the passage comprehension levels of students suddenly 
decreased in April 2020, but the rate of growth in passage comprehension was the same before and after April 2020.

Means of event level factors can be less intuitive to interpret. While the event level factor mean is -24.96 , the drop in points at pandemic onset is estimated at 7.00 as the estimated value takes into account the intercept, slope, and event. Thus, between the third and fourth time point, the slope factor contributes a 17.96-point increase while the even factor contributes a 24.96-point decrease, yielding the estimated 7.00-point decrease. We highly recommend calculating estimated values to ensure proper interpretation of LGM results (see excel spreadsheet with formulas in online supplemental material).

\section{Level and slope discontinuity latent growth models}

A final discontinuity model is the combination of both previous models. Accordingly, the level and slope discontinuity model estimates whether an event (e.g., onset of pandemic) is associated with changes in levels (i.e., a bump) as well as a different growth pattern (slope) preand post-event. In terms of data, a minimum of five time points is required (three time points per estimated slope).

Level and slope discontinuity, Example 4. In this example, our model simulates the hypothesis that sudden shifts in everyday routines at pandemic onset were associated with an abrupt drop in passage comprehension test scores, and scores increased at a slower pace during the pandemic than before the pandemic. To examine a level and slope discontinuity model with two linear slopes, the intercept set at the initial assessment, and the event occurring at the fourth of six assessments (see Figure 8), the loadings of the intercept and event level factors are constrained in the same manner as in the previous model (level discontinuity model). The loadings of the first slope construct are constrained to a linear pattern of 0 to 2 for the three 
assessments before the event, with zeros after the event. The loadings of the second slope construct are constrained to a linear pattern of 0 to 2 from the event onwards, with zeros before the event. Models in lavaan and Mplus return the same results. Growth factor means were significantly different from zero and estimated at $422.93(\mathrm{SE}=.17, \mathrm{p}<.001)$ for the intercept, $18.05(\mathrm{SE}=.11, \mathrm{p}<.001)$ for the first slope, $29.01(\mathrm{SE}=.06, \mathrm{p}<.001)$ for the event level, and $10.00(\mathrm{SE}=.11, \mathrm{p}<.001)$ for the second slope. These values indicate, as shown in Figure 9, an average level in passage comprehension of 422.93 points in October of Pre-K, with an average increase of 18.05 points per six months pre-pandemic onset, a drop of 7.09 points at pandemic onset, and an average increase of 10.00 points per six months post-pandemic onset. Thus, passage comprehension levels of students suddenly decreased in April 2020 and the rate of growth in passage comprehension being slower after April 2020 compared to before.

Here again, the event level factor mean is not straightforward in interpretation. While the event level factor mean is 29.01 , it corresponds to a drop in points at pandemic onset estimated at 7.09 as loadings at event onset are zero for both slopes, and thus only the intercept and event level factors contribute to the estimated value. Thus, the estimated value at the fourth assessment is 29.01 points higher than the estimated value at the first assessment, which also corresponds to 7.09 points less than the third time point. In other words, the positive value of the event level factor mean is due to the fact that passage comprehension points at pandemic onset in the spring of Kindergarten is higher than the value in the fall of pre-K, despite having decreased compared to the fall of Kindergarten, the last assessment pre-pandemic.

\section{Latent basis growth model}

A final model that may be useful for researchers to examine patterns of growth around the pandemic is the latent basis LGM (Grimm et al., 2016; sometimes referred to as the level and 
shape LGM, Little, 2013). These models freely estimate the shape of the slope. While latent basis LGMs are typically used as a basis of comparison for constrained models specifying the nature of the change function, they can also be used as a final model to best capture the actual shape of the slope. Accordingly, these models may be useful for capturing changes in growth such as those captured in discontinuity models when researchers do not have the minimum data collection waves to estimate these constrained models, such as in the example below. To freely estimate the shape of the slope, one of the loadings of the slope is fixed to zero (which also centers the intercept as with constrained models), and one of the loadings is fixed to a non-zero value, while the other loadings are freely estimated. The estimated loadings are interpreted to make conclusions about the shape of the slope.

Latent basis, Example 5. In this example, we use the same sample and model as for the slope discontinuity model above, but with students followed from April of Pre-K to October of Grade 1, yielding only four assessments. As mentioned above, to estimate a latent basis LGM, we need to fix one loading to 0 and one loading to a non-zero value. While in theory any nonzero value could be attributed to any loading, three main methods are used (see Figure 10). In a first method (Figure 10a), we fix the first and last loadings using the values that would be used for a linear LGM, in this case 0 and 3 . When using this method with our hypothetical data, the second loading is estimated at 1.20 and the third loading is estimated at 2.41, indicating that there is a non-linear trend. The exact nature of the change function is, however, difficult to interpret without calculating estimated values, which is remedied with the next two methods. The second method is to fix the first loading to 0 and the last loading to 1 (Figure 10b). When using this method, our second loading is estimated at 0.40 and our third loading is estimated at 0.80 . These loadings are interpreted as the proportion of the total change between Time 1 (loading of 
0 ) and Time 4 (loading of 1). Accordingly, they suggest that $40 \%$ of the total change occurred by the second timepoint and $80 \%$ of the total change occurred by the third timepoint. Another way of interpreting these numbers it that $40 \%$ of the change occurs between Time 1 and Time $2,40 \%$ occurs between Time 2 and Time 3, and 20\% occurs between Time 3 and Time 4 . The last option is to fix the first loading to 0 and second loading to 1 (Figure 10c). When using this method, our third loading is estimated at 2.00 and our third loading is estimated at 2.50. These loadings are interpreted in terms of the amount of change that occurred between Time 1 (loading of 0 ) and Time 2 (loading of 1). Accordingly, they suggest that the amount of change between Time 2 and Time 3 is the same as between Time 1 and Time 2 (difference in loadings both $=1$ ), but the amount of change between Time 3 and Time 4 is half of the previous timepoints (difference in loadings $=0.5$, which is coherent with the results from the previous method.

All three methods of setting the slope loadings return the same results. The three models return the same parameter estimates for the intercept $($ mean $=440.94, \mathrm{SE}=.21, \mathrm{p}<.001)$, but different parameters for the slope: model (a) mean $=14.98, \mathrm{SE}=.09, \mathrm{p}<.001$; model (b) mean $=$ 44.93, $\mathrm{SE}=.26, \mathrm{p}<.001 ;$ model $(\mathrm{c})$ mean $=18.04, \mathrm{SE}=.11, \mathrm{p}<.001)$. Using these values with the estimated loadings, however, yields the same estimated values for all three models (with slight differences due to rounding of loadings and mean values; see spreadsheet in online supplemental materials). Thus, while choices made in terms of fixing the loadings can influence ease of interpretation, they do not have an impact on the results. Furthermore, as can be seen in Figure 11, while we did not have enough time points to estimate a discontinuous LGM, the latent basis LGM allowed us to see that there seems to be a discontinuity around pandemic onset. 


\section{Estimating latent growth models after data collection disruptions}

When shutdowns to contain the spread of COVID-19 were implemented, many universities and research centers halted in-person data collections. Even after such disruptions, the LGMs presented above can be estimated, but slight modifications in the specification of the models may be necessary. In the remainder of this section, we present solutions for studies that had to cancel or delay data collection waves that had not yet started when shutdowns began, followed by solutions for studies that had started collecting data and had to cancel or delay the remainder of the data collection when shutdowns began. We provide examples by applying these disruptions to the simulated data of the slope discontinuity LGM presented above, with lavaan and Mplus syntaxes provided in online supplemental material.

\section{Disruption 1: Cancelled or delayed data collection wave}

For some studies, there may have been no current data collection when shutdowns began, but a data collection wave may have been planned during shutdowns, leading this data collection wave to be cancelled or delayed until re-openings. In this case, either there is no data for one of the planned data collection waves, or the time lag between some of the data collection waves are not those that were planned. Researchers that had planned to examine LGMs using equally spaced assessments can still estimate their LGMs using slight changes in coding strategies, where loadings are modified to properly represent the unequally spaced assessments that follow the data collection delay or cancellation. For example, in the linear LGM presented above, with six bi-annual assessments and linear slope loadings of $0,1,2,3,4,5$, cancelling the fourth assessment would yield loadings of $0,1,2,4,5$ and delaying the fourth assessment by three months would yield loadings of $0,1,2,3.5,4,5$. This can also be done for discontinuity models, as exemplified below. 
Cancelled wave, Example 6. In this hypothetical example based on the slope discontinuity model presented above, the planned data collection wave in April 2020 was cancelled. We can estimate the model in the exact same manner as with all the data collection points, but without the fourth assessment (see Figure 12). Here, the cancelled assessment corresponds to the knot point (i.e., common 0 for both slopes). Although the slope discontinuity model is usually depicted with a knot at a measurement time, the knot can also be between two measurement points. The knot point is then centered at the time where both slopes would have a common loading of zero, with the value corresponding to the point where the two estimated slopes reach each other (i.e., their crossover point). In our example, loading increments of 1.0 correspond to 6 months of time, and 6 months from the loading of -1.0 (October 2019, first slope) and 1.0 (October 2020, second slope) is April 2020, which centers the knot at that time. Results of this model (see Table 2) show that we would reach similar conclusions as if there had been no data collection cancellation.

Delayed wave, Example 7. In this hypothetical example, the planned data collection wave of April 2020 was delayed to July 2020, thus slope loadings have to be adapted to reflect the true time gap between assessments (see Figure 13). Thus, with the loading increments of 1.0 still corresponding to 6 months of time, the loading of the second slope on the fourth assessment is 0.5 as it is 3 months after the intended intercept (which here is the knot point). There is also a difference in loadings of 0.5 between the fourth and fifth assessment, which are now also 3 months apart due to the delay. Results of this model (see Table 2) show that we would reach similar conclusions as if there had been no data collection delay. 


\section{Disruption 2: Partially cancelled data collection wave}

In some cases, data collection waves already under way may have been shut down and researchers either made the decision not to complete the data collection after re-openings or were unable to resume, leading to an incomplete data collection for that wave. In this case, data is available for participants for whom data was collected before shutdowns and missing for the subset of participants that had not yet answered. This missing data will be either missing completely at random (MCAR) or missing at random (MAR), meaning that this disruption can be remedied using modern missing data treatments (i.e., multiple imputation or full information maximum likelihood estimation; Lang \& Little, 2018; Rioux \& Little, 2021).

MCAR is a truly random process, where the reason for a missing data point is unrelated to the observed variables, missing values, and all unmeasured variables, and thus the participants with missing data are not different to those with non-missing data. This process is the most rare, except when implementing a planned missing data design (Rioux et al., 2020), but may be a tenable hypothesis for some COVID-19 disruptions. Indeed, if the order in which participants partook in the data collection wave that had to be partially cancelled due to shutdowns was completely random, the underlying missing data process may be MCAR. MAR is a systematic, predictable, process where the missing values can be predicted by other variables in the observed data (Enders, 2010; Seaman et al., 2013). Accordingly, if some participant characteristics were associated with the order of participation (e.g., neighborhood, age, grade, date of last assessment), the missing data process would be MAR. In both the MCAR and MAR cases, missing data treatments can recover power and provide unbiased results. A third missing data mechanism, missing not at random (MNAR), is a systematic, but unpredictable, process where the missing data points are predicted by the missing value itself (Enders, 2010; Seaman et al., 
2013). When data are MNAR, estimates are biased even with the use of modern missing data treatments, as there is no way to recover the missing information. However, in the case of COVID -19-related cancellations in data collection, the data are not missing because of the missing values themselves, but rather because of outside factors. Even if participants that had not yet participated before the shutdowns are systematically different from participants who answered before shutdowns, these differences did not cause them to drop out of that data collection wave. Accordingly, these differences follow a MAR process and can be accounted for in the missing data treatments.

If the data are in theory MAR and the covariate related to the process is not included in the missing data treatment, the analysis would follow a MNAR pattern and be biased. Thus, auxiliary variables related to the MAR mechanism must be used to obtain unbiased estimates (Enders, 2008; Howard et al., 2015). Auxiliary variables are variables that are not of interest for the research questions, but that predict missingness, and can be included in both multiple imputation and FIML analyses. Importantly, missing data treatments require some information about the associations between variables with missing data and other variables in the dataset to be available, which is why these methods cannot be used to include a variable from completely cancelled data collection waves in the model specification (see solutions in Disruption 1 above).

As previously mentioned, multiple imputation and FIML are two options to recover power and obtain unbiased estimates when missing data is MAR or MCAR. Multiple imputation is a two-step approach, where (1) missing values are estimated and substituted with a predicted value, which is done multiple times to create multiple complete (imputed) datasets, and (2) analyses are run on each of the imputed datasets and then aggregated. Comprehensive theory and guidance for multiple imputation can be found in van Buuren (2018) and Enders (2010). FIML is 
a model-based approached that uses the available data (complete and incomplete) to produce the most accurate parameter estimates and standard errors in a single step. FIML is implemented by most SEM software, making its use more frequent than multiple imputation for LGMs. Comprehensive theory and guidance on FIML can be found in Enders (2010) and Raykov (2005).

Partially cancelled, Example 8. In this hypothetical example, our April data collection was underway when shutdowns occurred, and half our students are missing this data collection wave. With missing data MCAR, we used FIML to estimate our model parameters in the presence of these missing observations without additional modifications to our models. In a second hypothetical situation, the order in which participants were contacted for follow-up led to students from lower socio-economic status (SES) to be more likely to have been among students for whom the April data collection was cancelled, thus SES was included as an auxiliary variable to account for this MAR mechanism. In both the MCAR and MAR cases, all estimates show that we reach similar conclusions as if there had been no data collection cancellation (see Table 2).

\section{Disruption 3: Partially delayed data collection wave}

Other data collection waves already under way may have been shut down but were able to resume after re-openings. Accordingly, for the same planned data collection wave, some participants would have data before shutdowns (i.e., before the pandemic), and the remaining participants would have data after shutdowns (i.e., during the pandemic). One solution is to treat the data as a multiple group LGM (see Grimm et al., 2016, chapter 6) and assign the delayed group the corresponding lagged slope loading (as done in Disruption 1).

Partially delayed, Example 9. In this hypothetical example, half of our sample was able to complete the assessment before shutdowns in April 2020, but the other half had the same 
assessment delayed to July 2020. A multiple group model was estimated, with Group 1 having the same slope loadings as the model without disruptions (Slope discontinuity, Example 2) and Group 2 having the slope loadings from Delayed wave, Example 7. Through this specification, all estimates show that we would reach similar conclusions as if there had been no data collection cancellation (see Table 2). Furthermore, if this is of interest, this method can also allow one to see whether results differ according to the time at which data was collected through multigroup difference testing (see Bollen \& Curran, 2006, Chapter 6; Little, 2013, Chapter 7).

\section{Conclusion}

Following the onset of the COVID-19 pandemic, discontinuities in expected developmental patterns can be expected and many researchers collecting in-person longitudinal data had to cancel or delay data collection waves that were either underway or beginning during shutdowns. We presented several LGMs that allow the examination of growth discontinuities and offered several statistical solutions that can be utilized for analysing these models following COVID-19-related disruptions in data collection. While appropriate solutions will vary for each study depending on its design, questions, and the nature of disruptions in data collection, we highlight straightforward statistical solutions for the most common pandemic-related disruptions in data collection. In general, we show that while planned data analyses may have to be revised, in many cases researchers interested in examining growth processes can proceed in examining their original research questions. Furthermore, in many cases, the longitudinal nature of the data will allow examination of some pandemic-related effects. This ability is an important strength of longitudinal studies that were ongoing before the pandemic as they can observe development before, during, and after the pandemic, with results that can generalize to more environmental and historical conditions. 


\section{References}

Bernal, J. L., Cummins, S., \& Gasparrini, A. (2017). Interrupted time series regression for the evaluation of public health interventions: a tutorial. International Journal of Epidemiology, 46(1), 348-355. https://doi.org/10.1093/ije/dyw098

Bollen, K. A., \& Curran, P. J. (2006). Latent curve models: A structural equation perspective. Wiley.

Chou, C. P., Yang, D. Y., Pentz, M. A., \& Hser, Y. I. (2004). Piecewise growth curve modeling approach for longitudinal prevention study. Computational Statistics \& Data Analysis, 46(2), 213-225. https://doi.org/10.1016/s0167-9473(03)00149-x

Coulombe, P., Selig, J. P., \& Delaney, H. D. (2016). Ignoring individual differences in times of assessment in growth curve modeling. International Journal of Behavioral Development, 40(1), 76-86. https://doi.org/10.1177/0165025415577684

Enders, C. K. (2008). A note on the use of missing auxiliary variables in full information maximum likelihood-based structural equation models. Structural Equation Modeling: A Multidisciplinary Journal, 15(3), 434-448. https://doi.org/10.1080/10705510802154307

Enders, C. K. (2010). Applied Missing Data Analysis. The Guildford Press.

Flora, D. B. (2008). Specifying piecewise latent trajectory models for longitudinal data. Structural Equation Modeling: A Multidisciplinary Journal, 15(3), 513-533. https://doi.org/10.1080/10705510802154349

Grimm, K., \& Marcoulides, K. (2016). Individual change and the timing and onset of important life events: Methods, models, and assumptions. International Journal of Behavioral Development, 40(1), 87-96. https://doi.org/10.1177/0165025415580806 
Grimm, K. J., Ram, N., \& Estabrook, R. (2016). Growth Modeling: Structural Equation and Multilevel Modeling Approaches. The Guilford Press.

Gupta, S., \& Jawanda, M. K. (2020). The impacts of COVID-19 on children. Acta Paediatrica, 109(11), 2181-2183. https://doi.org/10.1111/apa.15484

Howard, W. J., Rhemtulla, M., \& Little, T. D. (2015). Using Principal Components as Auxiliary Variables in Missing Data Estimation. Multivariate Behavioral Research, 50(3), 285-299. https://doi.org/10.1080/00273171.2014.999267

Isiordia, M., \& Ferrer, E. (2018). Curve of Factors Model: A Latent Growth Modeling Approach for Educational Research. Educational and Psychological Measurement, 78(2), 203-231. https://doi.org/10.1177/0013164416677143

Kohli, N., \& Harring, J. R. (2013). Modeling Growth in Latent Variables Using a Piecewise Function. Multivariate Behavioral Research, 48(3), 370-397. https://doi.org/10.1080/00273171.2013.778191

Lang, K. M., \& Little, T. D. (2018). Principled Missing Data Treatments. Prevention Science, 19(3), 284-294. https://doi.org/10.1007/s11121-016-0644-5

Lange, S. J., Ritchey, M. D., Goodman, A. B., Dias, T., Twentyman, E., Fuld, J., Schieve, L. A., Imperatore, G., Benoit, S. R., Kite-Powell, A., Stein, Z., Peacock, G., Dowling, N. F., Briss, P. A., Hacker, K., Gundlapalli, A. V., \& Yang, Q. H. (2020). Potential Indirect Effects of the COVID-19 Pandemic on Use of Emergency Departments for Acute LifeThreatening Conditions - United States, January-May 2020. Morbidity and Mortality Weekly Report, 69(25), 795-800. https://doi.org/10.15585/mmwr.mm6925e2

Little, T. D. (2013). Longitudinal Structural Equation Modeling. The Guildford Press. 
Marchetti, D., Fontanesi, L., Mazza, C., Di Giandomenico, S., Roma, P., \& Verrocchio, M. C. (2020). Parenting-Related Exhaustion During the Italian COVID-19 Lockdown. Journal of Pediatric Psychology, 45(10), 1114-1123. https://doi.org/10.1093/jpepsy/jsaa093

Masonbrink, A. R., \& Hurley, E. (2020). Advocating for Children During the COVID-19 School Closures. Pediatrics, 146(3), Article e20201440. https://doi.org/10.1542/peds.2020-1440

Mehta, P. D., \& West, S. G. (2000). Putting the individual back into individual growth curves. Psychological Methods, 5(1), 23-43. https://doi.org/10.1037//1082-989x.5.1.23

Muthén, L. K., \& Muthén, B. O. (1998-2017). Mplus User's Guide (8th ed.). Muthén \& Muthén. Pellegrini, M., Ponzo, V., Rosato, R., Scumaci, E., Goitre, I., Benso, A., Belcastro, S., Crespi, C., De Michieli, F., Ghigo, E., Broglio, F., \& Bo, S. M. N. (2020). Changes in Weight and Nutritional Habits in Adults with Obesity during the "Lockdown" Period Caused by the COVID-19 Virus Emergency. Nutrients, 12(7), Article 2016. https://doi.org/10.3390/nu12072016

Peugh, J. L., Beal, S. J., McGrady, M. E., Toland, M. D., \& Mara, C. (2020). Analyzing discontinuities in longitudinal count data: A multilevel generalized linear mixed model. Psychological Methods. Advance online publication. https://doi.org/10.1037/met0000347

R Core Team. (2020). R: A Language and Environment for Statistical Computing. R Foundation for Statistical Computing. R-project.org

Rajkumar, R. P. (2020). COVID-19 and mental health: A review of the existing literature. Asian Journal of Psychiatry, 52, Article 102066. https://doi.org/10.1016/j.ajp.2020.102066

Raykov, T. (2005). Analysis of longitudinal studies with missing data using covariance structure modeling with full-information maximum likelihood. Structural Equation Modeling: A Multidisciplinary Journal, 12(3), 493-505. https://doi.org/10.1207/s15328007sem1203_8 
Rioux, C., Lewin, A., Odejimi, O. A., \& Little, T. D. (2020). Reflection on modern methods: planned missing data designs for epidemiological research. International Journal of Epidemiology, 49(5), 1702-1711. https://doi.org/10.1093/ije/dyaa042

Rioux, C., \& Little, T. D. (2021). Missing data treatments in intervention studies: What was, what is, and what should be. International Journal of Behavioral Development, 45(1), 5158. https://doi.org/10.1177/0165025419880609

Roos, L. E., Salisbury, M., Penner-Goeke, L., Cameron, E. E., Protudjer, J. L. P., Giuliano, R., Afifi, T., \& Reynolds, K. (2021). Supporting Families to Protect Child Health: Parenting Quality and Household Needs During the COVID-19 Pandemic. Plos One, 16(5), Article e0251720. https://doi.org/10.1371/journal.pone.0251720

Rosseel, Y. (2012). lavaan: An R Package for Structural Equation Modeling. Journal of Statistical Software, 48(2), 1-36. https://doi.org/10.18637/jss.v048.i02

RStudio Team. (2020). RStudio: Integrated Development Environment for R. RStudio, Inc. https://rstudio.com

Seaman, S., Galati, J., Jackson, D., \& Carlin, J. (2013). What is meant by "Missing at Random"? Statistical Science, 28(2), 257-268. https://doi.org/10.1214/13-sts415

Singer, J. D., \& Willett, J. B. (2003). Applied Longitudinal Data Analysis: Modeling Change and Event Occurrence. Oxford University Press.

Skibbe, L. E., Grimm, K. J., Bowles, R. P., \& Morrison, F. J. (2012). Literacy Growth in the Academic Year Versus Summer From Preschool Through Second Grade: Differential Effects of Schooling Across Four Skills. Scientific Studies of Reading, 16(2), 141-165. https://doi.org/10.1080/10888438.2010.543446 
Tomfohr-Madsen, L. M., Racine, N., Giesbrecht, G. F., Lebel, C., \& Madigan, S. (2021). Depression and anxiety in pregnancy during COVID-19: A rapid review and metaanalysis. Psychiatry Research, 300, article 113912. https://doi.org/10.1016/j.psychres.2021.113912

van Buuren, S. (2018). Flexible imputation of missing data (2nd ed.). CRC Press.

van de Schoot, R., Schmidt, P., De Beuckelaer, A., Lek, K., \& Zondervan-Zwijnenburg, M. (2015). Editorial: Measurement Invariance. Frontiers in Psychology, 6(1064). https://doi.org/10.3389/fpsyg.2015.01064

Widaman, K. F., Ferrer, E., \& Conger, R. D. (2010). Factorial Invariance Within Longitudinal Structural Equation Models: Measuring the Same Construct Across Time. Child Development Perspectives, 4(1), 10-18. https://doi.org/10.1111/j.17508606.2009.00110.x 
Table 1. Overview of LGMs presented as solutions for COVID-19-related discontinuities in change and disruptions in longitudinal data collection

\begin{tabular}{|c|c|c|}
\hline Model, Example \# & Problem & Solution \\
\hline \multicolumn{3}{|l|}{ Discontinuities in change } \\
\hline Slope discontinuity, Example 2 & Change in rate of change after pandemic onset & Estimate a second slope after a knot point \\
\hline Level discontinuity, Example 3 & Sudden level bump after pandemic onset & Add an event factor \\
\hline Level and slope discontinuity, Example 4 & $\begin{array}{l}\text { Level bump and change in rate of change after } \\
\text { pandemic onset }\end{array}$ & Estimate a second slope and add an event factor \\
\hline Latent basis, Example 5 & $\begin{array}{l}\text { Too few assessments to estimate level and/or slope } \\
\text { discontinuity }\end{array}$ & Freely estimate slope loadings \\
\hline \multicolumn{3}{|l|}{ Disruptions in longitudinal data collection } \\
\hline Cancelled wave, Example 6 & Measurement occasions not equidistant & Change the loading of the slope factor \\
\hline Delayed wave, Example 7 & Measurement occasions not equidistant & Change the loading of the slope factor \\
\hline Partially cancelled wave, Example 8 & Wave with incomplete data & Use missing data treatments \\
\hline Partially delayed wave, Example 9 & $\begin{array}{l}\text { Wave with participants measured at two different } \\
\text { times }\end{array}$ & Use multiple group LGM \\
\hline
\end{tabular}


Table 2. Results of slope discontinuity model without data collection disruptions and for each disruption condition

\begin{tabular}{llllllll}
\hline & & Knot & & Slope 1 & & Slope 2 \\
Model & Condition & Mean & SE & Mean & SE & Mean & SE \\
\hline No disruptions (Example 2) & & 477.00 & .36 & 18.03 & .10 & 8.93 & .13 \\
Cancelled wave, Example 6 & & 477.01 & .36 & 18.03 & .10 & 8.92 & .13 \\
Delayed wave, Example 7 & & 476.98 & .36 & 18.03 & .10 & 8.97 & .12 \\
Partially cancelled, Example 8 & MCAR & 477.05 & .36 & 18.05 & .10 & 8.90 & .12 \\
Partially cancelled, Example 8 & MAR & 476.95 & .47 & 18.01 & .11 & 8.87 & .15 \\
Partially delayed, Example 9 & Group 1 & 477.40 & .51 & 18.17 & .15 & 9.18 & .18 \\
Partially delayed, Example 9 & Group 2 & 477.59 & .49 & 18.15 & .14 & 8.94 & .21 \\
\hline
\end{tabular}

Note. Parameter estimates all significant at $\mathrm{p}<.001$. 


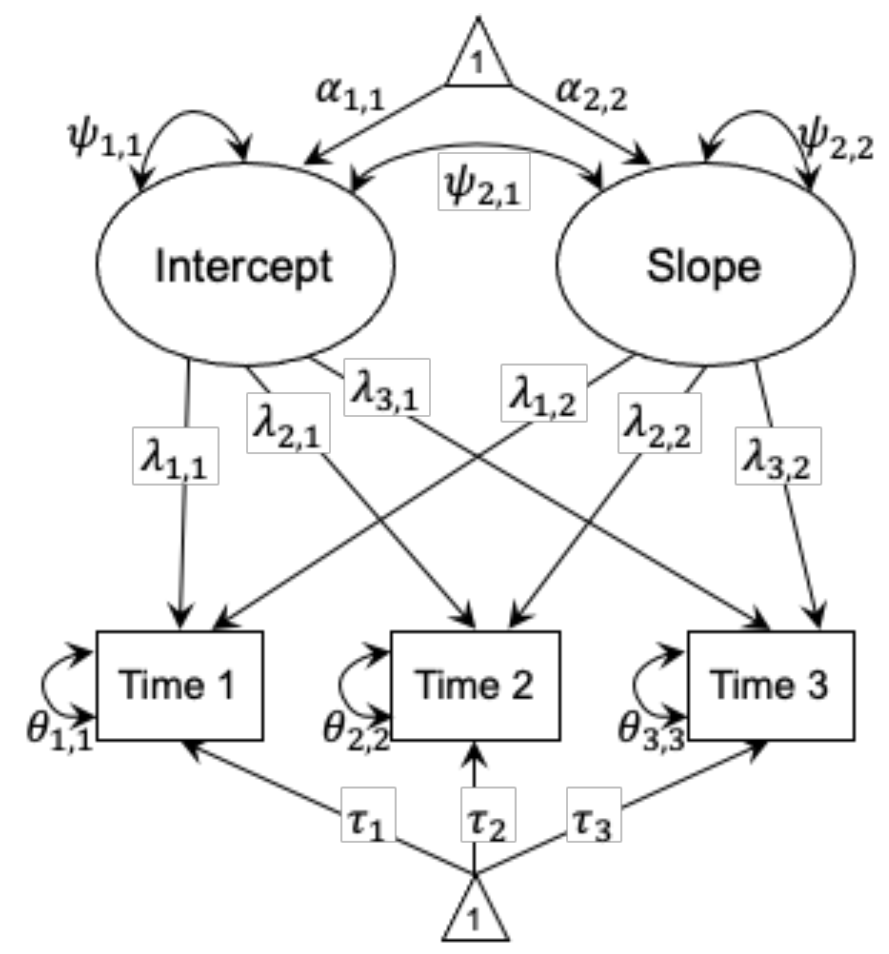

Figure 1. Path diagram with all parameters for a basic latent growth model. $\alpha=$ latent construct mean; $\psi=$ variance and covariance; $\lambda=$ factor loading; $\theta=$ indicator residual variance; $\tau=$ residual variance mean (fixed to 0 ). 


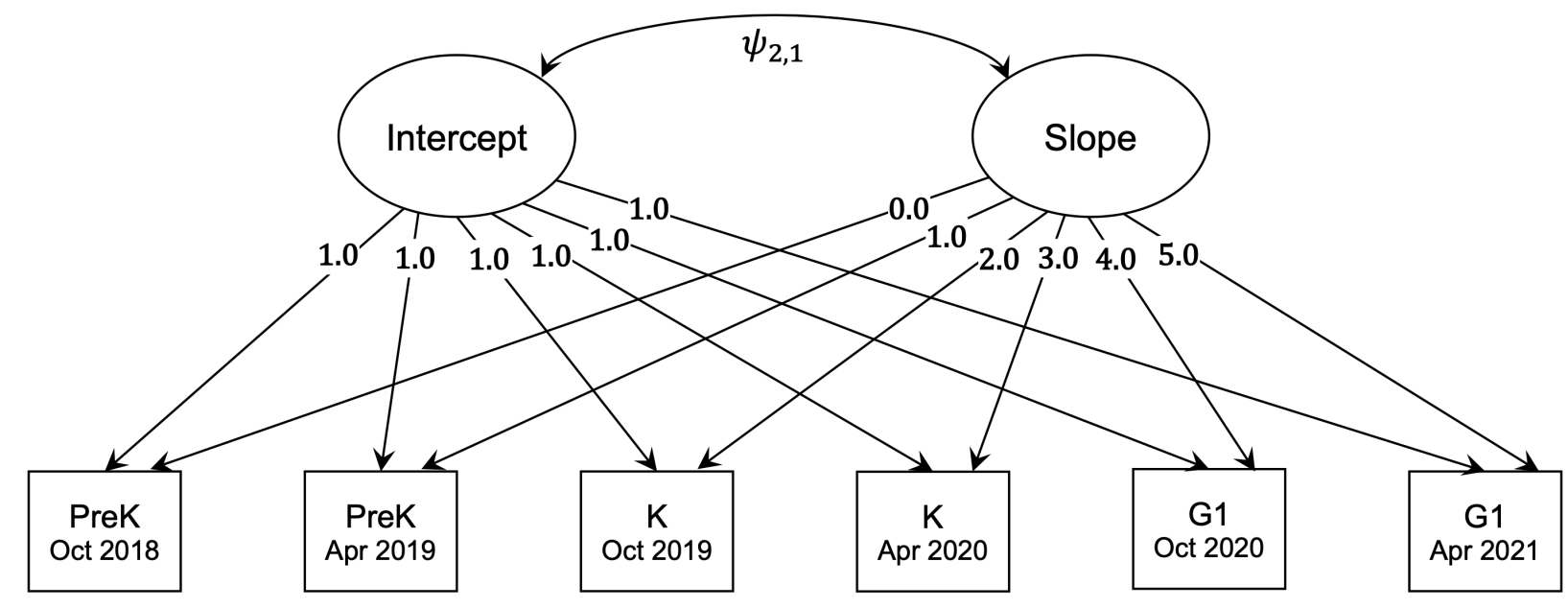

Figure 2. Linear LGM, Example 1; Linear growth model with participants followed from one year before kindergarten to Grade 1 over six bi-annual assessments. PreK = One year before kindergarten; $\mathrm{K}=$ Kindergarten; $\mathrm{G1}$ = Grade 1 . Mean structure not included for reading ease.

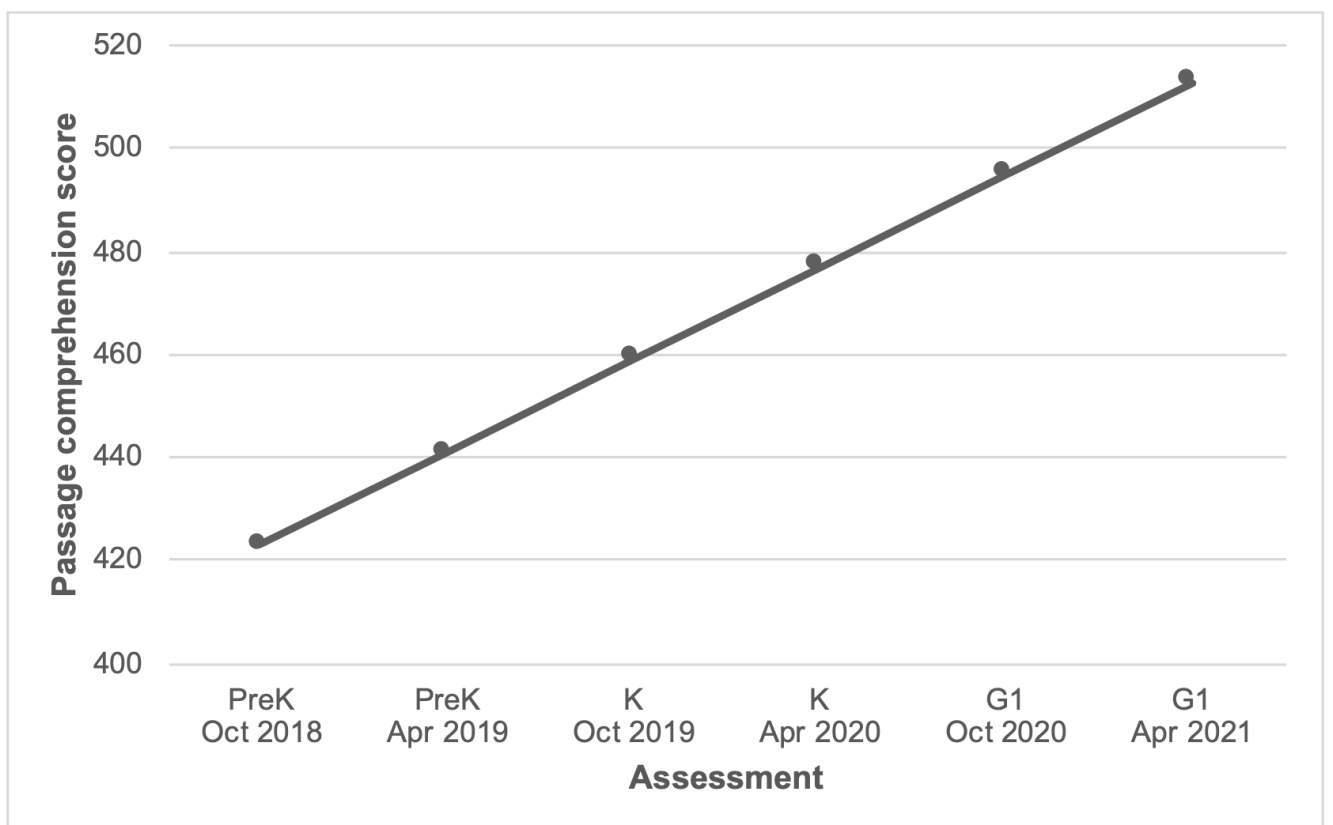

Figure 3. Linear LGM, Example 1; Estimated average growth trajectory from the linear LGM. PreK = One year before kindergarten; $\mathrm{K}=$ Kindergarten; $\mathrm{G1}=$ Grade 1. 


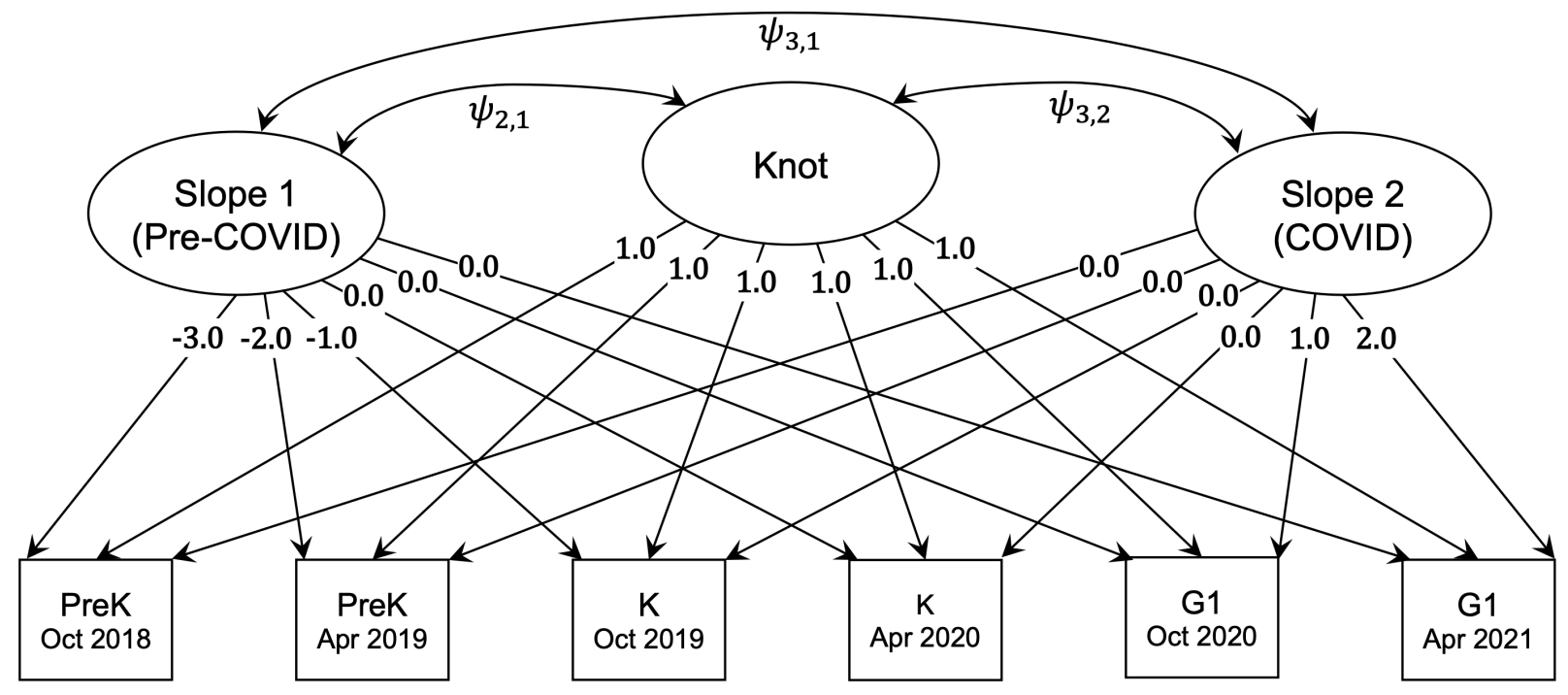

Figure 4. Slope discontinuity, Example 2; Path diagram of the estimated slope discontinuity LGM. PreK = One year before kindergarten; $\mathrm{K}=$ Kindergarten; G1 = Grade 1. Mean structure not included for reading ease.

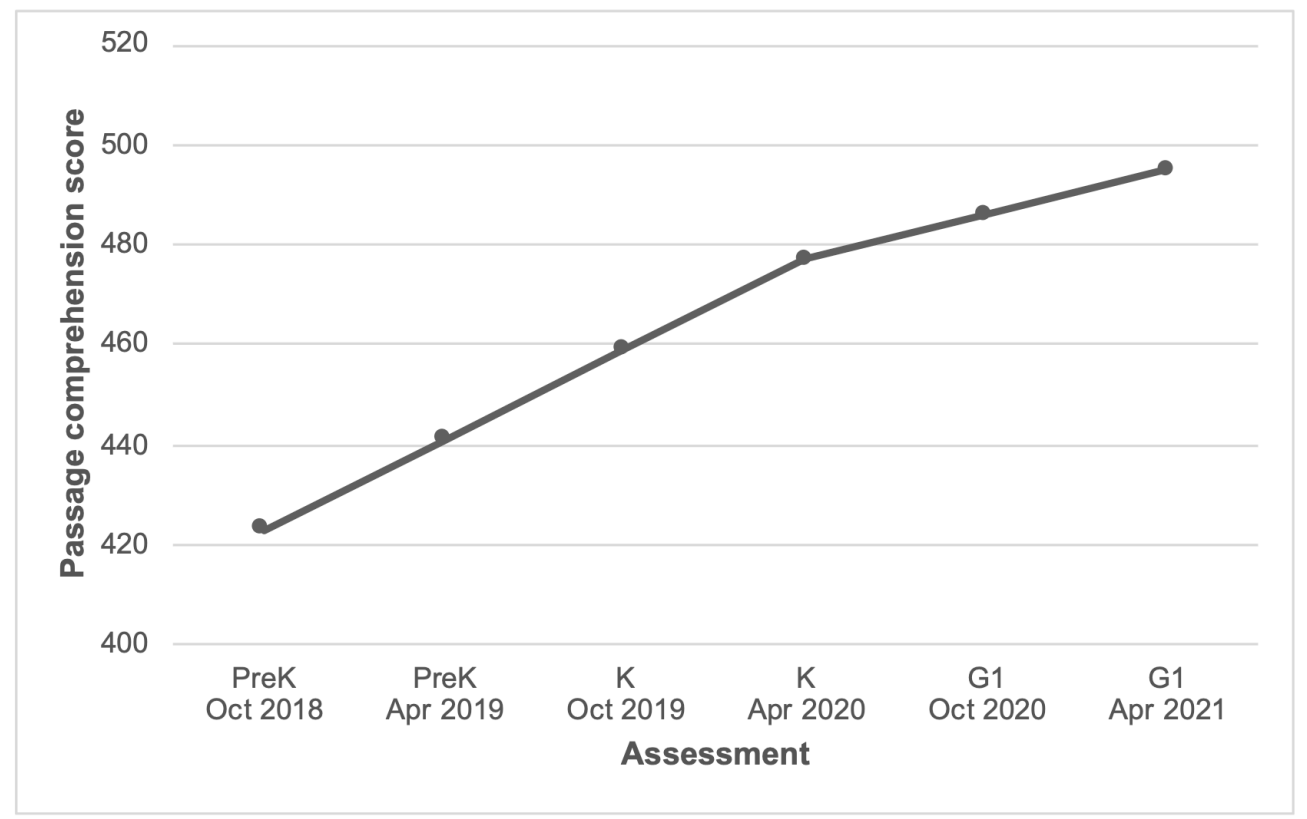

Figure 5. Slope discontinuity, Example 2; Estimated average growth trajectory from the slope discontinuity LGM. PreK = One year before kindergarten; $\mathrm{K}=$ Kindergarten; G1 = Grade 1. 


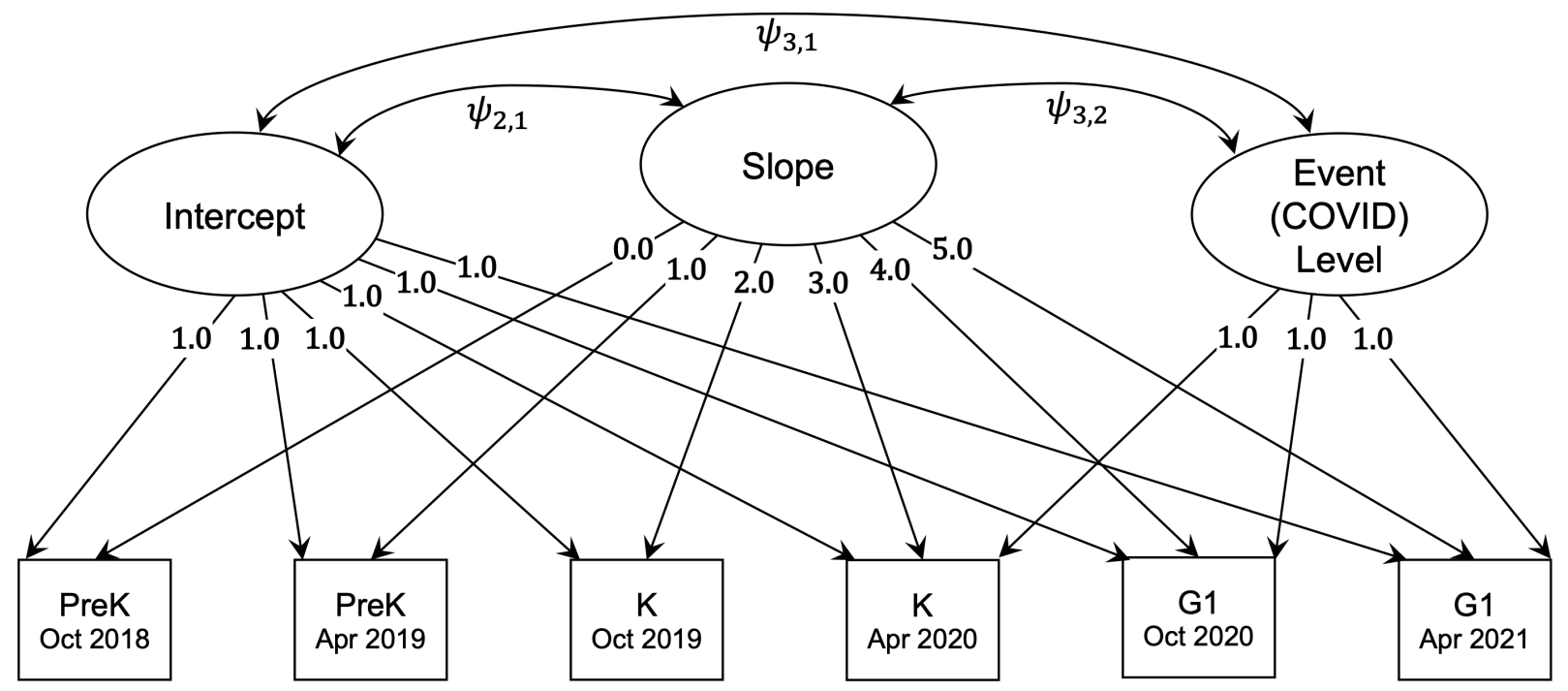

Figure 6. Level discontinuity, Example 3; Path diagram of the estimated level discontinuity LGM. PreK = One year before kindergarten; $K=$ Kindergarten; $\mathrm{G} 1$ = Grade 1 . Mean structure not included for reading ease.

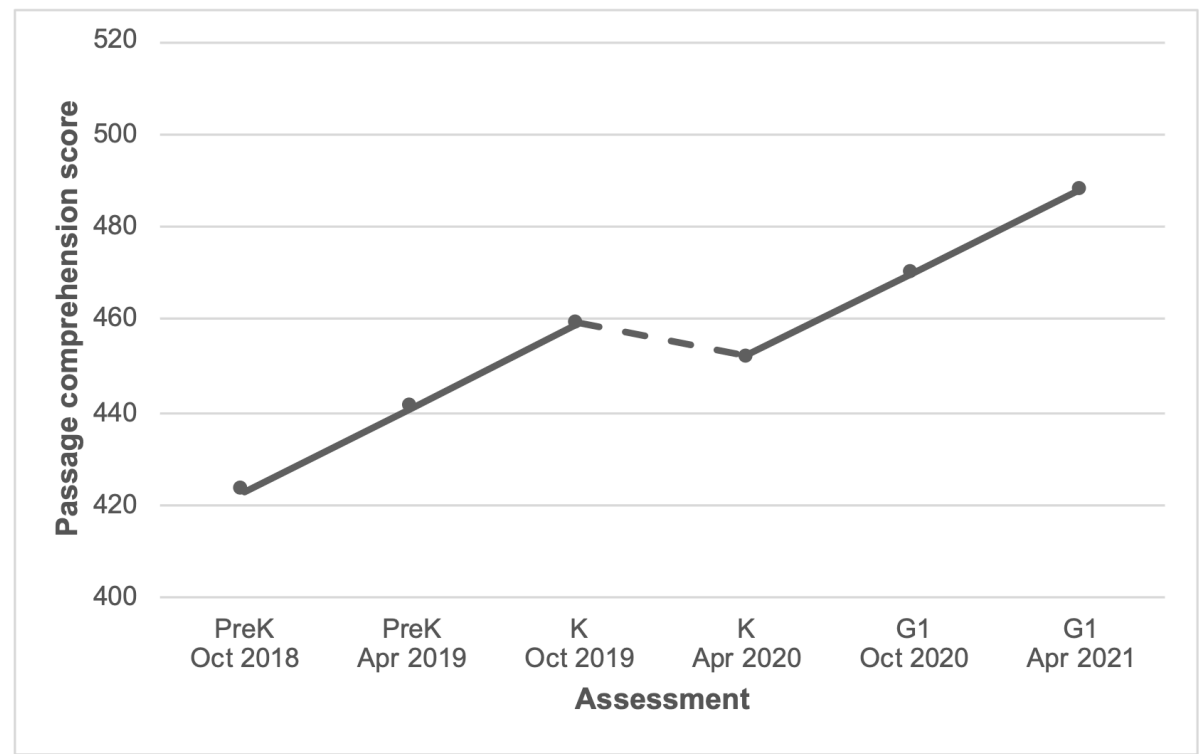

Figure 7. Level discontinuity, Example 3; Estimated average growth trajectory from the level discontinuity LGM. PreK = One year before kindergarten; $\mathrm{K}=$ Kindergarten; $\mathrm{G1}=$ Grade 1 . 


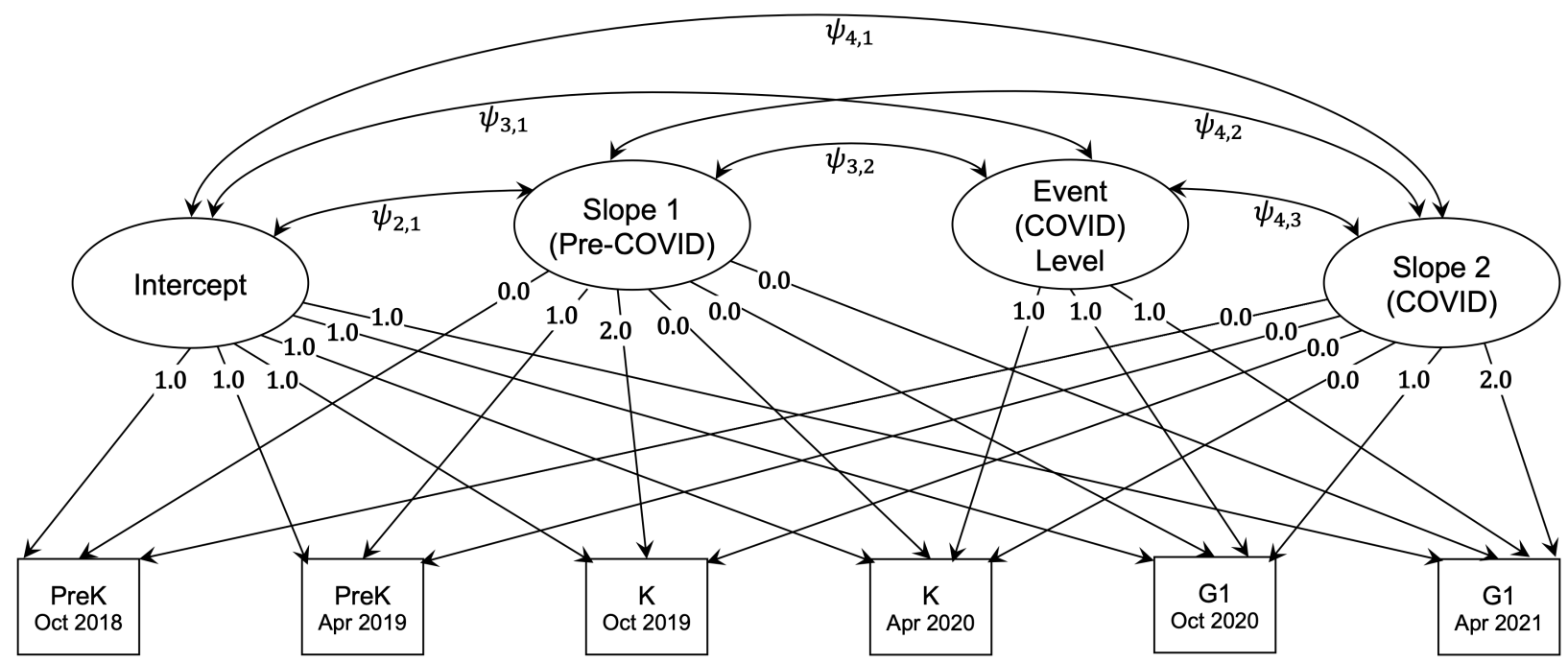

Figure 8. Level and slope discontinuity, Example 4; Path diagram of the estimated level and slope discontinuity LGM. PreK = One year before kindergarten; K = Kindergarten; G1 = Grade 1. Mean structure not included for reading ease.

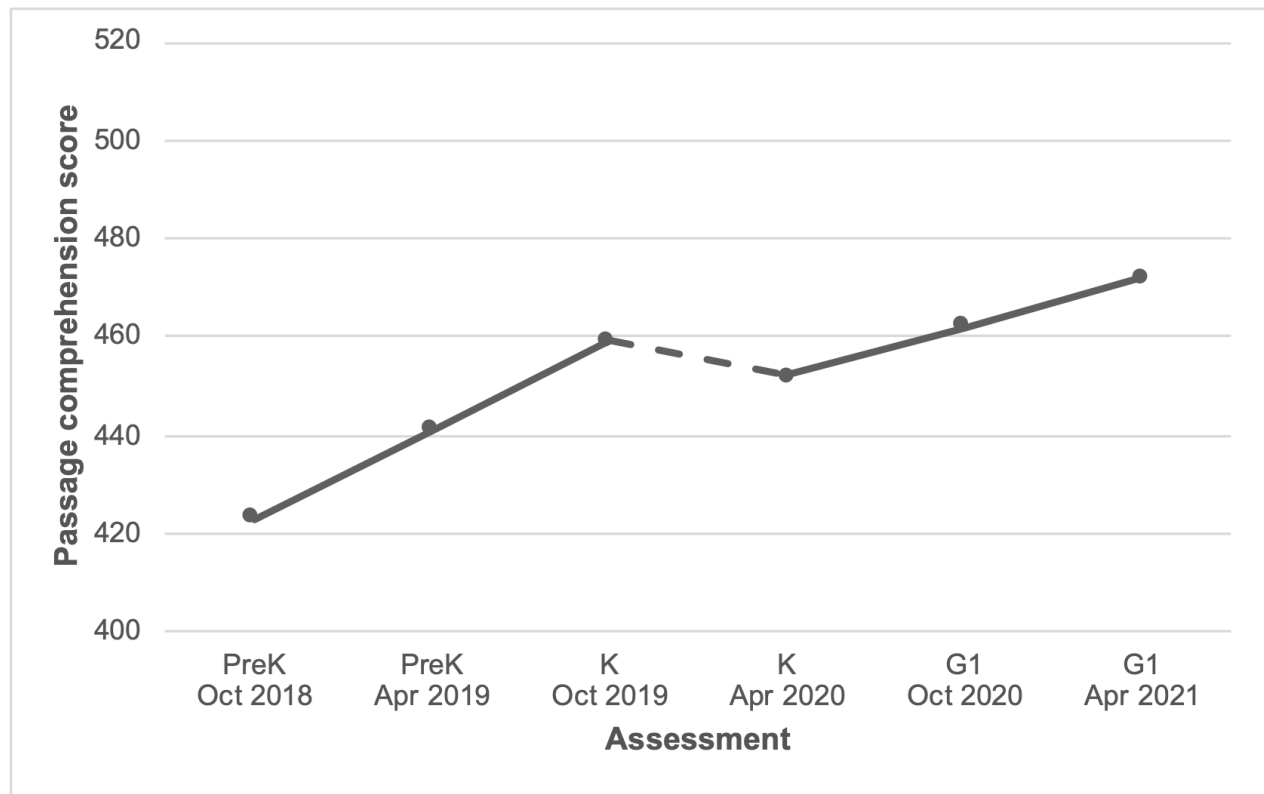

Figure 9. Level and slope discontinuity, Example 4; Estimated average growth trajectory from the level and slope discontinuity LGM. PreK = One year before kindergarten; K = Kindergarten; G1 = Grade 1 . 


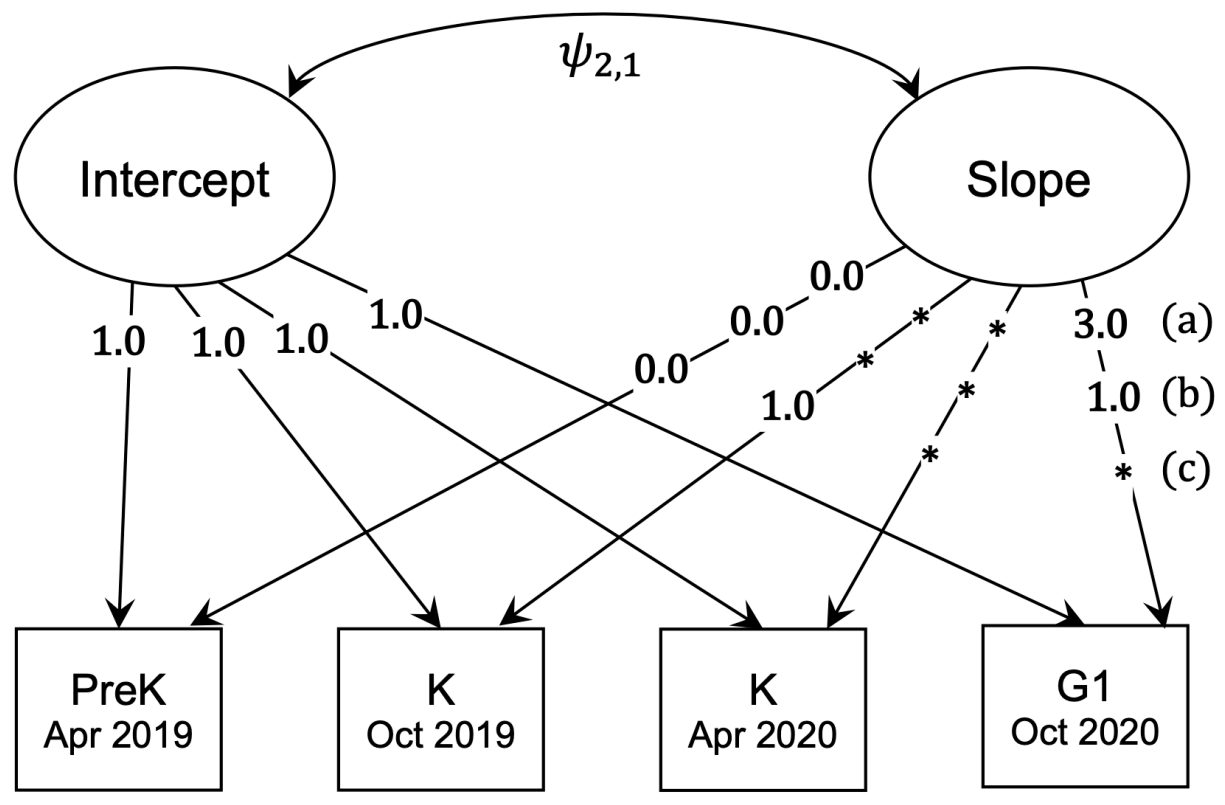

Figure 10. Latent basis, Example 5; Path diagram of the estimated latent basis LGMs. (a), (b), and (c) show different loading constraints used. PreK = One year before kindergarten; $\mathrm{K}=$ Kindergarten; G1 = Grade 1. Mean structure not included for reading ease.

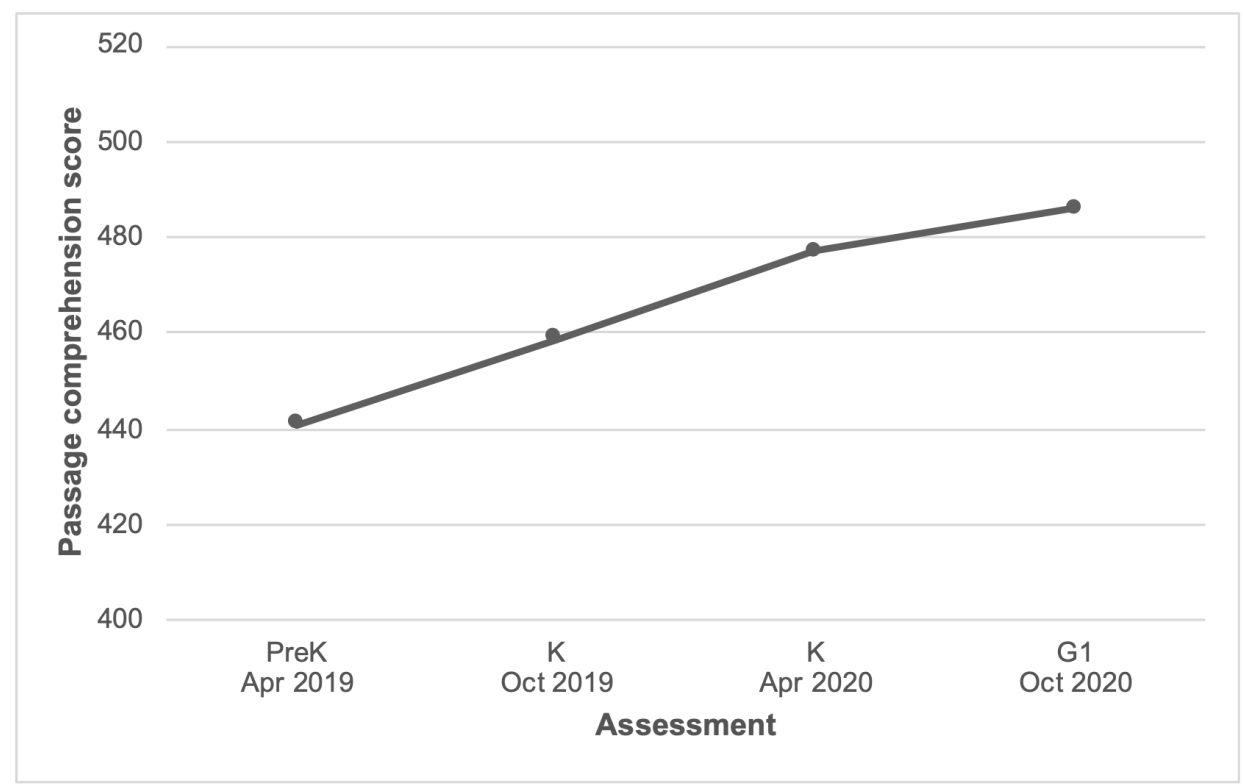

Figure 11. Latent basis, Example 5; Estimated average growth trajectory from the latent basis LGM. PreK = One year before kindergarten; K = Kindergarten; G1 = Grade 1. 


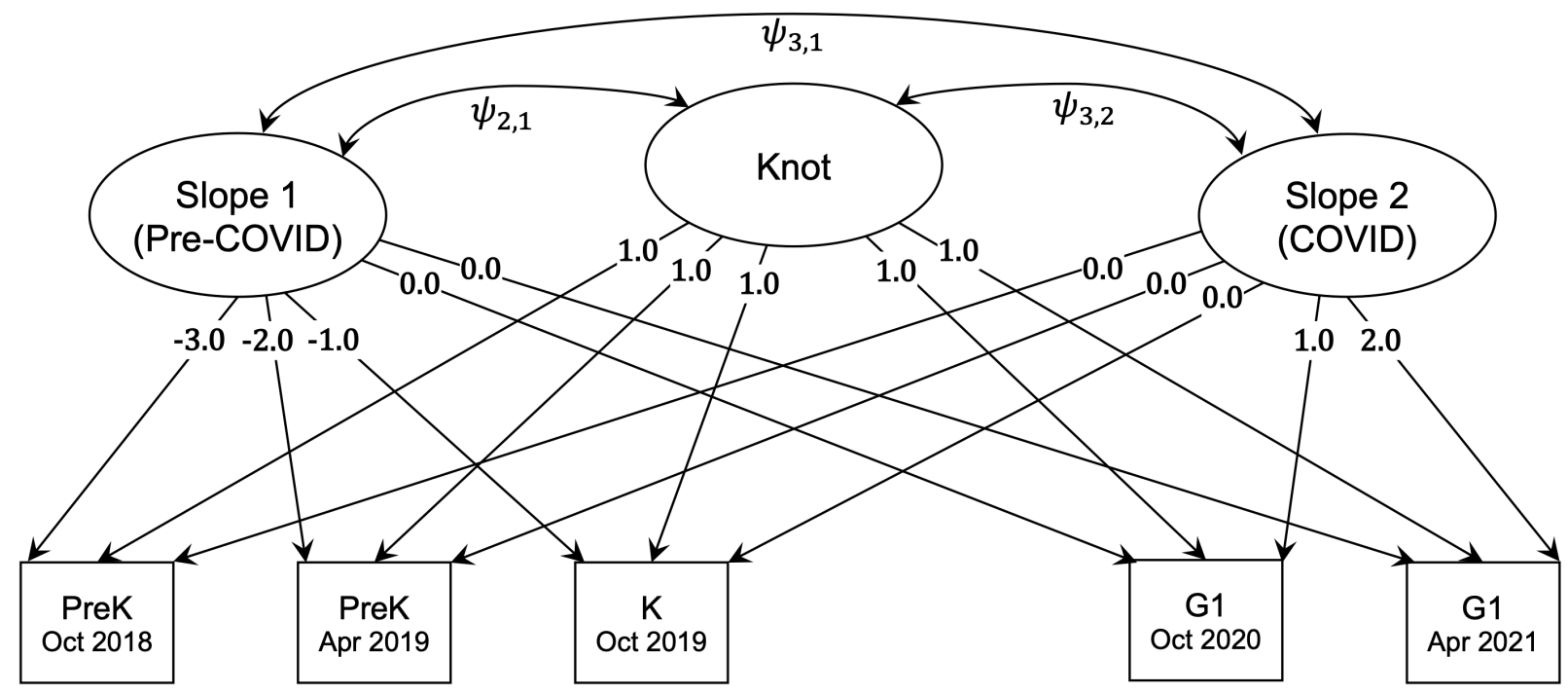

Figure 12. Cancelled wave, Example 6; Path diagram of the estimated slope discontinuity LGM after the cancellation of the fourth data collection wave (04/2020). PreK = One year before kindergarten; $\mathrm{K}=$ Kindergarten; $\mathrm{G1}=$ Grade 1. Mean structure not included for reading ease.

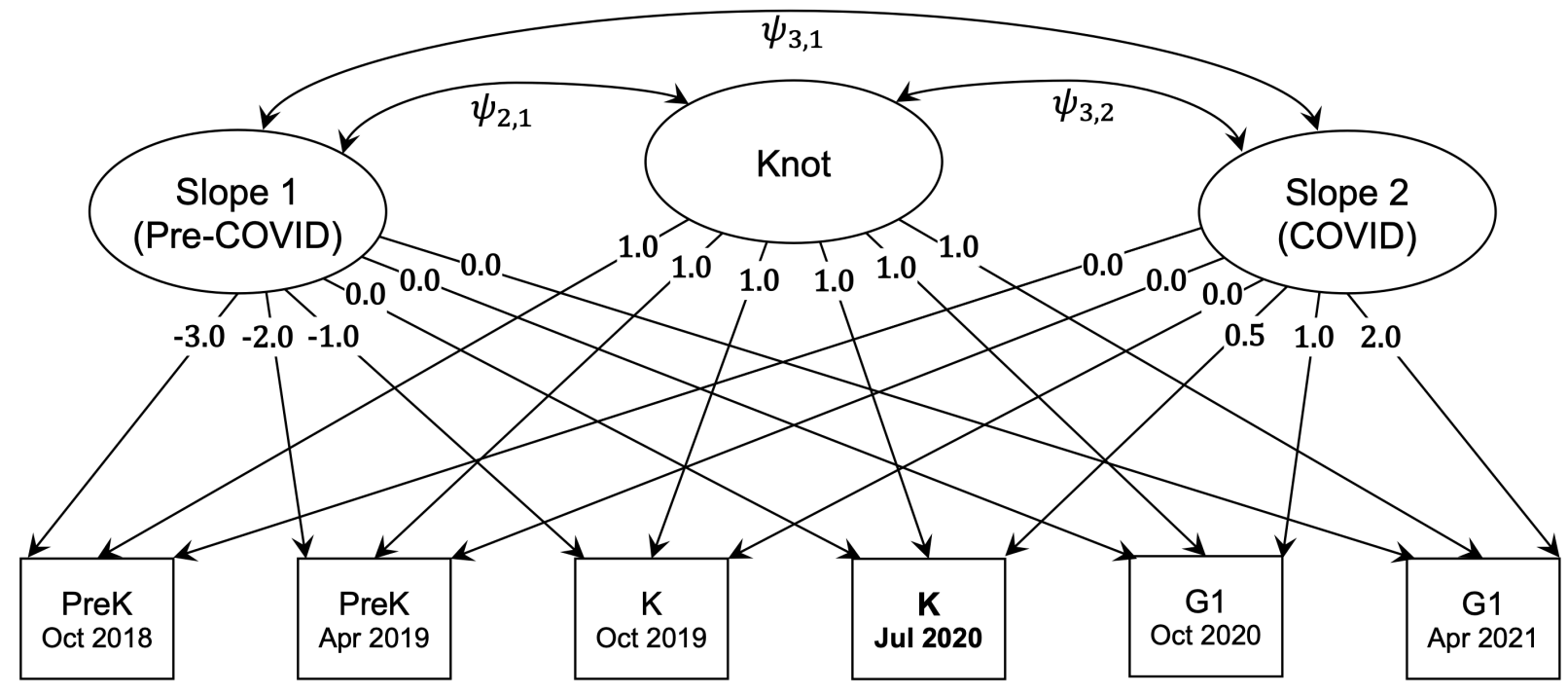

Figure 13. Delayed wave, Example 7; Path diagram of the estimated slope discontinuity LGM after delay of the fourth data collection wave (04/2020) by three months (to 07/2020). PreK= One year before kindergarten; $K=$ Kindergarten; $G 1$ = Grade 1. Mean structure not included for reading ease. 